\title{
Positive-parity Baryon Spectra on Isotropic Lattice
}

\section{Tanjib Khan*}

Department of Physics, William \& Mary, Williamsburg, Virginia, USA.

E-mail: mkhano1@email.wm. edu

\section{David Richards}

Thomas Jefferson National Accelerator Facility, Newport News, Virginia, USA.

E-mail: dgr@jlab.org

\section{Frank Winter}

Thomas Jefferson National Accelerator Facility, Newport News, Virginia, USA.

E-mail: fwinterejlab.org

We present a calculation of the low-lying spectrum for the positive-parity Delta and Nucleon using the distillation approach applied on an isotropic lattice at a pion mass of around $360 \mathrm{MeV}$. We use a non-relativistic basis of three-quark operators, together with so-called hybrid-type operators. The results are compared with lattice calculations at higher pion masses on an anisotropic lattice. The effect of varying the distillation space on the calculation is explored with the aim of obtaining stable results with a minimal number of distillation eigenvectors.

The 36th Annual International Symposium on Lattice Field Theory - LATTICE2018

22-28 July, 2018

Michigan State University, East Lansing, Michigan, USA.

\footnotetext{
* Speaker.
} 


\section{Introduction}

While the excited meson spectrum has been explored extensively, the study of the baryon spectrum is more limited, and the most extensive studies have focused on anistropic lattices[1, 2], where the spatial and temporal lattice spacings are different. Nevertheless, these works, using three-quark interpolating operators, have provided important insights into the nature of the excited spectrum: a counting of states at least as rich as that of the quark model, and richer than that of a straightforward point-like diquark/quark picture, and strong indications of additional positiveparity states beyond those of the quark model that are "hybrid" in nature, where gluonic degrees of freedom play an essential role [1].

Despite the advantage of the anisotropic formulation, notably in enabling a finer resolution of the correlation functions, it carries disadvantages, most notably the more complicated operator renormalization needed both for hadron structure, and for the transition matrix elements to excited states. In this work, we study the use of an isotropic action for determining the lowlying positive-parity baryon spectrum, and compare with the earlier, anisotropic studies; a study of nucleon charges using the same ensemble and computational framework, namely distillation [3], appears elsewhere [4]. In particular, we explore the calculation of the positive-parity nucleon and delta masses with somewhat lighter quark masses, at a finer spatial lattice spacing, thus probing the robustness of spin identification and that of identifying hybrid baryons. Finally, we explore the sensitivity of our calculation to the rank of the distillation basis.

\section{Computational Strategy \& Lattice Parameters}

We are interested in the low-lying regions of the spectra, so interpolators are chosen in such a way that they yield the correlators that have been found to have a large overlap with the low-lying, positive-parity spectrum. Most of the interpolators are constructed with only the upper components of the Dirac spinor, in the Dirac basis; they are referred to as non-relativistic operators, with the derivatives acting as the orbital angular momentum between the quarks. There is further class of interpolators formed from the commutation of two gauge-covariant derivatives acting on the same quark field; such operators correspond to the chromomagetic components of the gluonic fieldstrength tensor and are referred to as "hybrid" operators.

As the calculations are done on discretized lattice, the baryons with higher total angular momenta J, are subduced from continuous Hilbert space into discrete lattice irreps. containing multiple J. As a result, we have multiple interpolators corresponding to the same continuum operator for the cases of higher total angular momenta, with those interpolators lying in different lattice irreps. The numbers of interpolators used in our calculation are listed in Table 1.

The operators created directly from the fields of the lattice lagrangian have significant overlap with all energies of the spectrum making the extraction of the lightest elements of the spectrum difficult. To address this problem, a linear operator is applied on the quark fields on appropriate time-slices and operators are built from those "smeared" fields. In this work, the smearing method used is known as Distillation [3]. The distillation operator on time-slice is defined as:

$$
\square_{x y}(t)=\sum_{k=1}^{N_{D}} v_{x}^{(k)}(t) v_{y}^{(k) \dagger}(t) \quad \Rightarrow \quad \square(t) \equiv V_{D}(t) \otimes V_{D}^{\dagger}(t)
$$




\begin{tabular}{|c|c|c|c|}
\hline J & irrep. (dim) & No of Interpolators $(\Delta)$ & No of Interpolators $(\mathrm{N})$ \\
\hline$\frac{1}{2}$ & $G_{1}(2)$ & 3 & 7 \\
$\frac{3}{2}$ & $H(4)$ & 5 & 7 \\
$\frac{5}{2}$ & $G_{2}(2) \oplus H(4)$ & $2+2$ & $4+4$ \\
$\frac{7}{2}$ & $G_{1}(2) \oplus G_{2}(2) \oplus H(4)$ & $1+1+1$ & $1+1+1$ \\
\hline
\end{tabular}

Table 1: Baryons of total angular momenta subduced into lattice irreps.

where $v_{x}^{(k)}(t)$ are eigenvectors of the three-dimensional Laplacian on times slice $t$, ordered by the size of the corresponding eigenvalue, and $N_{D}$ is the number of eigenvectors used to construct the distillation operator.

The distillation method enables us to break down the calculation into smaller independent pieces, namely the construction of the operators and quark propagator, the latter of which can be reused in other calculations. Secondly, it enables extended operators both at source and sink to be computed at no additional cost in terms of quark propagator, enabling the full panoply of the variational method to be employed. Finally, the method performs spatial sums, or momentum projections, at both the source and sink time-slices, thus increasing the statistical precision significantly. The method does, however, have a significant drawback; the number of eigevectors $N_{D}$ needed to determine the low-energy spectrum is anticipated to grow as the spatial volume, while the cost of constructing the correlators as $\simeq N_{D}^{4}$ for the operators considered here. Thus an important aim is to make the distillation space sufficiently small that the low-energy spectrum can be calculated reliably while keeping the computational cost affordable.

We employ a Wilson-Clover fermion action with periodic boundary conditions in space, and anti-periodic in time, and a tree-level tadpole-improved Symanzik gauge action. We use degenerate up and down quarks, and a heavier strange quark. To smooth the short distance fluctuations of the gauge field, we applied "stout" smearing [5] on the gauge field on both spatial links and temporal ones. We note that an important difference between these lattices and the anisotropic ones is the use of smearing in the temporal direction, precluding a physical transfer matrix, which may yield some distortion of the spectrum. The lattice is of extent $32^{3} \times 64$, with lattice spacing $a=0.098$ fm using $\mathrm{w}_{0}$ to set the scale, yielding a pion mass of $356 \mathrm{MeV}$. The ensemble comprises 351 gauge configurations, and the so-called "perambulators" \& "elementals" are computed for a total of $N_{D}=64$ eigenvectors from five different times sources $t_{\mathrm{m}}=0,8,12,16$ and 32. To extract the energy spectra and the operator overlaps, we use the the variational method [6] which involves solving a generalized eigenvalue equation (GEV).

\section{Results}

The general format of the operators is written as,

$$
\left(\mathrm{B}_{\Sigma_{\mathrm{F}}} \otimes\left(\mathrm{S}^{\mathrm{PS}}\right)_{\Sigma_{\mathrm{S}}}^{n} \otimes \mathrm{D}_{\mathrm{L}, \Sigma_{\mathrm{D}}}^{[d]}\right)^{\mathrm{J}}
$$

where B is the flavor of the particle involved and $\Sigma_{\mathrm{F}}$ is the associated permutational symmetry, $\mathrm{S}$ is the spin of the particle, $P_{S}$ is the parity with $\Sigma_{\mathrm{S}}$ permutational symmetry. There can be multiple 
possible combinations of upper and lower components of Dirac spinors resulting in the same spin, parity and symmetry, and these are distinguished by the superscript $n$. The third element is the derivative factor indicating the number of gauge-covariant derivatives applied to the quark fields by $d$ with permutational symmetry $\Sigma_{\mathrm{D}}$. The derivatives are combined to give objects which transforms invariantly under rotations with angular momentum $L$.

The operator overlap factors are used to identify the spin of a state $[2,7]$. As each interpolator used in the calculation carries an essence of the continuum spin from which it is subduced, we would expect an interpolator subduced from spin $\mathrm{J}$, to have large overlaps only onto states corresponding to the same continuum spin J, and we therefore compare the Z-values obtained independently in each irrep. In Figure 1, the overlaps for different interpolators in the $\mathrm{H}_{\mathrm{g}}$ and $\mathrm{G}_{2 \mathrm{~g}}$

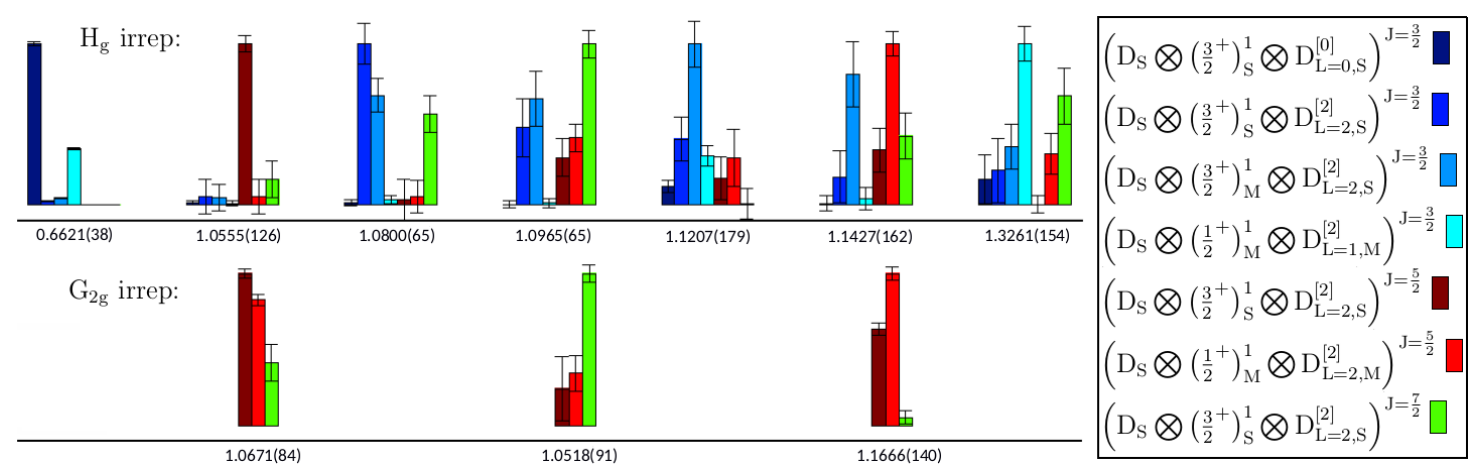

Figure 1: Histogram plot of operator overlaps, $\mathrm{Z}$ for Delta for irreps. $H_{g}$ (top), $G_{2 g}$ (bottom). Along the $x$-axes, the energy levels are given in lattice unit.

irreps. for the case of the Delta are shown in the upper and lower panels respectively. The overlaps are normalized so that for a given interpolator, the largest value across all the states is equal to unity, and the numbers along the $x$ axes are the energies of the states in lattice units. The overlaps corresponding to operators with spins $\mathrm{J}=\frac{3}{2}, \mathrm{~J}=\frac{5}{2}$, and from $\mathrm{J}=\frac{7}{2}$ are shown in blue, red and green, respectively.

From Table 1, we see that the positive parity states with continuum spin, $\mathrm{J}=\frac{5}{2}$ subduce onto $H_{g} \& G_{2 g}$ irreps. and for $J=\frac{7}{2}$, the states subduce onto $H_{g}, G_{1 g} \& G_{2 g}$ irreps. So, we should get similar overlaps for operators subduced onto the $\mathrm{H}_{\mathrm{g}} \& \mathrm{G}_{2 \mathrm{~g}}$ irreps. for these states. Figure 1 shows that our expectations are indeed satisfied. In $\mathrm{H}_{\mathrm{g}}$ irrep., the states with dominating contributions from the operators with $\mathrm{J}=\frac{5}{2}$ and $\mathrm{J}=\frac{7}{2}$ have the energy within $4 \%$ of that corresponding states in $\mathrm{G}_{2 \mathrm{~g}}$ irrep.; we do not show data for the $G_{1 g}$ irrep. for reasons of space, but the results are found to be consistent.

Our calculations show that the method of spin identification exploited in the anisotropic calculations is equally applicable to our calculation performed on isotropic lattices. Perhaps this is not surprising, since the spatial lattice spacing is finer, leading to a smaller breaking of the rotational symmetry that lies at the heart of the spin-identification technique.

We now examine the sensitivity of the calculated spectra to the dimension of the distillation space. Based on our expectations for volume scaling, we would anticipate the distillation space for the lattice used in our calculation should be around 225; we get satisfactory results while using $N_{D}=64$. Thus we now try reducing the distillation space further to see how the spectrum changes. 
In Figure 2, the spectra of the Delta particle in $\mathrm{Hg}$ irrep. are plotted for different values of $N_{D}$.

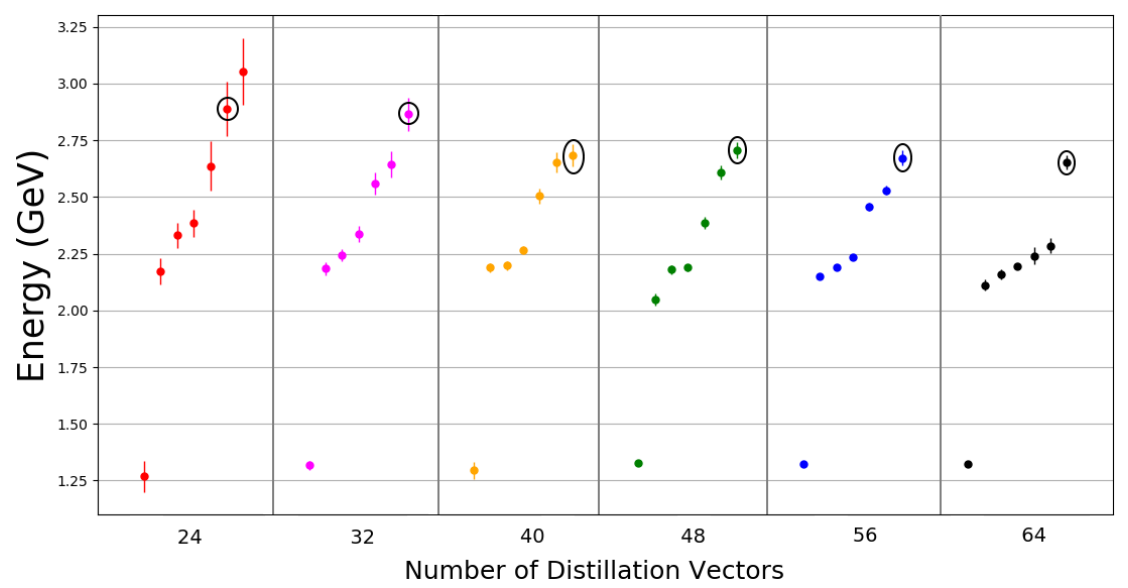

Figure 2: Effect of varying distillation space on Delta spectra in Hg irrep. States with dominant overlap of hybrid operators are marked by black circles.

Clearly as we reduce the distillation space, the spectrum starts to spread and the energy levels tend to have more uncertainty. Below $N_{D}=48$, the spectrum starts to deteriorate significantly and below $N_{D}=32$, it becomes almost unrecognizable changing the order of the hybrid state. The effects of reducing distillation space are much more prominent for the higher-excited states. For the lowerexcited states, and for the states identified as "hybrids", the results are relatively stable, but it is clear that a larger number of distillation vectors will be necessary to explore the highest states in the spectrum.

\section{Discussion}

Our results for the spectra for the positive-parity Delta and Nucleon are shown in Figures 3 and 4 respectively, and compared with the earlier work on an anistropic lattice [1]. In both plots, the energies are shown in $\mathrm{GeV}$ using the lattice spacing as determined in their respective analyses, and the $\mathrm{x}$-axes denote the spin $\mathrm{J}=\frac{1}{2}, \frac{3}{2}, \frac{5}{2}, \frac{7}{2}$, as identified above. For the Delta, in Figure 3, we can see that the energy spectra are qualitatively similar, with a cluster of energies around $2.2 \mathrm{GeV}$ and $2.3 \mathrm{GeV}$ respectively. As the pion mass decreases, the energy levels get closer to each other and an overall downward shift of all the energy levels is observed.

In [1], for $\mathrm{J}=\frac{5}{2}$ and $\mathrm{J}=\frac{7}{2}$, a joint fit is done to the principal correlators corresponding to the same operator but subduced into different irreps. resulting in only one mass per operator. We do not perform such a procedure here, but rather quote energy levels in each irrep. separately. Ideally, the energy levels from the same operator should have the same value, but due to the discretization effects, they differ from each other. We find that the corresponding energy levels for $\mathrm{J}=\frac{5}{2}$ and $\mathrm{J}=\frac{7}{2}$ have very close values showing the robustness of the method as applied on our relatively finer isotropic lattice.

In Figure 4, the energy spectrum of the nucleon for pion mass $356 \mathrm{MeV}$ is compared with that of pion mass $396 \mathrm{MeV}$. The qualitative behavior is similar to that of the Delta, with the spectrum compressing and shifting downward as pion mass decreases. 


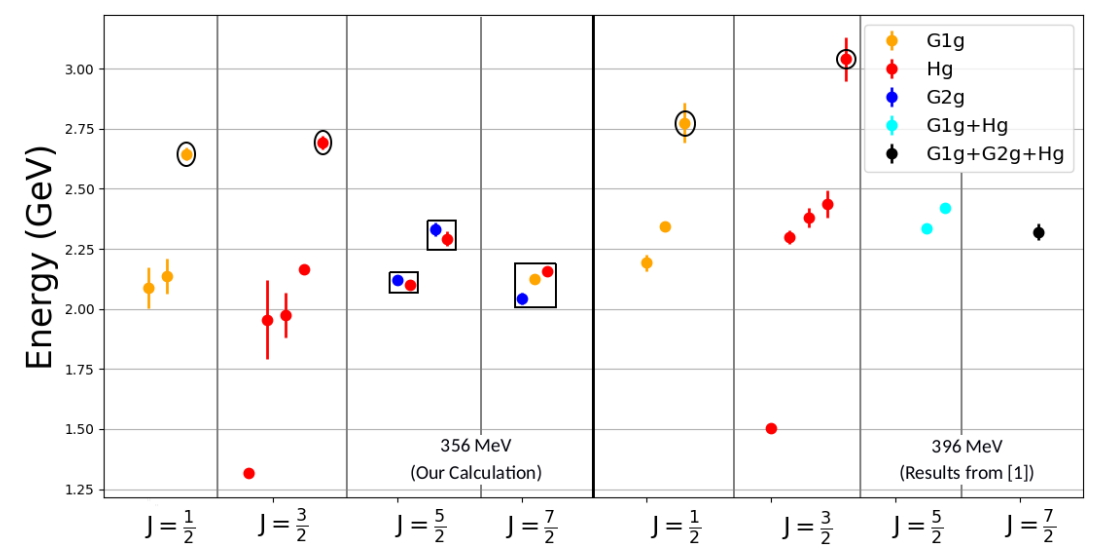

Figure 3: Energy Spectrum of Delta at $\mathrm{M}_{\pi}=356 \mathrm{MeV}$ (left), $396 \mathrm{MeV}$ (right). The energy levels corresponding to the same operator but subduced into different irreps. are boxed-in.

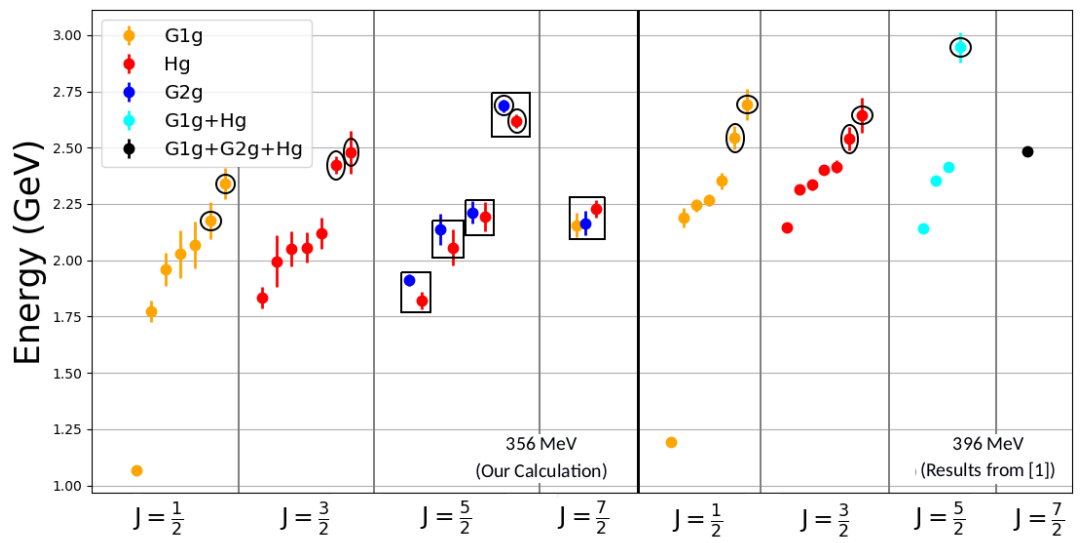

Figure 4: Energy Spectrum of Nucleon at $\mathrm{M}_{\pi}=356 \mathrm{MeV}$ (left), $396 \mathrm{MeV}$ (right). The energy levels corresponding to the same operator but subduced into different irreps. are boxed-in.

\section{Conclusion \& Future Work}

The low-lying spectra of baryons extracted here show remarkable resemblance to the previous calculations employing an anisotropic lattice. Even though the same set of operators were used, the actions are quite different, notably in the work described here the gauge fields of the action are smeared not only in the spatial directions but also in the temporal direction, in contrast to the anisotropic calculation. We are easily able to identify so-called "hybrid" states out of a dense spectrum of $q q q$ states supporting the robustness of the approach, despite the possibility of mixing strongly with $q q q$ states of the same $J^{P}$ and the lack of "exotic" quantum numbers that simplify their identification in the meson sector. We should emphasize, however, that this work is incomplete in that only three-quark operators, and not operators expected to couple to scattering states, are included in the basis. The extension of the calculation to lighter values of the pion mass is in progress. Our future effort will also include analyzing the energy spectra with non-zero momenta which will in turn be important in exploring the nature of hadron structure and in calculating quantities like Parton Distribution Functions, Transverse Momentum Dependent Distributions and 
Generalized Parton Distributions. Our approach deals with the single-hadron operators only. Further, this is a prerequisite to the inclusion of multi-hadron operators in the basis which will enable a realistic calculation of the spectrum [8].

\section{Acknowledgment}

We thank Jozef Dudek for useful discussions on the physics of hybrid baryons and for the use of his fitting codes. TK is grateful to Archana Radhakrishnan and Christopher Johnson for illuminating discussions regarding the calculation techniques. This research used resources of the Oak Ridge Leadership Computing Facility, and of National Energy Research Scientific Computing Center, which are DOE Office of Science User Facilities, operated under Contracts DE-AC0500OR22725 and DE-AC02-05CH11231 respectively.

This material is based on work supported by the U.S. Department of Energy, under contract DE-AC05-06OR23177. Computations for this work were carried out in part on facilities of the USQCD Collaboration, which are funded by the Office of Science of the U.S. Department of Energy, using Chroma [9] and QUDA[10].

\section{References}

[1] J. J. Dudek and R. G. Edwards, Hybrid Baryons in QCD, Phys. Rev. D85 (2012) 054016 [1201.2349].

[2] R. G. Edwards, J. J. Dudek, D. G. Richards and S. J. Wallace, Excited state baryon spectroscopy from lattice QCD, Phys. Rev. D84 (2011) 074508 [1104.5152].

[3] Hadron Spectrum collaboration, M. Peardon, J. Bulava, J. Foley, C. Morningstar, J. Dudek, R. G. Edwards et al., A Novel quark-field creation operator construction for hadronic physics in lattice QCD, Phys. Rev. D80 (2009) 054506 [0 905 .2160].

[4] C. Egerer, D. Richards and F. Winter, Controlling Excited-State Contributions with Distillation in Lattice QCD Calculations of Nucleon Isovector Charges $g_{S}^{u-d}, g_{A}^{u-d}, g_{T}^{u-d}, 1810.09991$.

[5] C. Morningstar and M. J. Peardon, Analytic smearing of SU(3) link variables in lattice QCD, Phys. Rev. D69 (2004) 054501 [hep-lat/ 0311018 ].

[6] J. J. Dudek, R. G. Edwards, N. Mathur and D. G. Richards, Charmonium excited state spectrum in lattice QCD, Phys. Rev. D77 (2008) 034501 [0 707 . 4162].

[7] J. J. Dudek, R. G. Edwards, M. J. Peardon, D. G. Richards and C. E. Thomas, Highly excited and exotic meson spectrum from dynamical lattice QCD, Phys. Rev. Lett. 103 (2009) 262001 [0909.0200].

[8] C. W. Andersen, J. Bulava, B. Hörz and C. Morningstar, Elastic I=3/2 p-wave nucleon-pion scattering amplitude and the $\Delta(1232)$ resonance from $N_{f}=2+1$ lattice $Q C D$, Phys. Rev. D97 (2018) 014506 [1710.01557].

[9] SCIDAC, LHPC, UKQCD collaboration, R. G. Edwards and B. Joo, The Chroma software system for lattice QCD, Nucl. Phys. Proc. Suppl. 140 (2005) 832 [hep-lat/ 0409003 ].

[10] M. A. Clark, R. Babich, K. Barros, R. C. Brower and C. Rebbi, Solving Lattice QCD systems of equations using mixed precision solvers on GPUs, Comput. Phys. Commun. 181 (2010) 1517 [0911.3191]. 